\title{
A eficiência técnica dos produtores leiteiros no Uruguai
}

\author{
Technical efficiency of dairy producers in Uruguay \\ Magela Cavalleri Ferrari¹ (D), Marcelo José Braga ${ }^{1}$ (D), \\ ${ }^{1}$ Universidade Federal de Viçosa (UFV), Viçosa (MG), Brasil. E-mails: magelacavalleri@gmail.com; \\ mjbraga.ruralprosper@gmail.com,
}

Como citar: Cavalleri Ferrari, M., \& Braga, M. J. (2021). A eficiência técnica dos produtores leiteiros no Uruguai. Revista de Economia e Sociologia Rural, 59(2), e221319. https://doi.org/10.1590/1806-9479.2021.221319

Resumo: O objetivo deste trabalho foi analisar a eficiência técnica dos produtores de leite no Uruguai, com base nos dados do Censo Geral Agropecuário, correspondente ao exercício produtivo 2010/2011. Empregou-se a abordagem da análise de fronteiras estocásticas, obtendo-se a medida da eficiência técnica média, assim como de cada estabelecimento. Os resultados mostraram que os produtores leiteiros do Uruguai operam, em média, aos 79,9\% da sua capacidade, o que mostra que muitos deles poderiam ainda melhorar seus rendimentos com a tecnologia disponível. Além disso, os resultados mostram que a assistência técnica e a utilização de silos reduzem possíveis ineficiências.

Palavras-chave: eficiência técnica, fronteiras estocásticas, produção leiteira.

\begin{abstract}
In the work, an estimation and analysis of the technical efficiency of the dairy producers in Uruguay was carried out, based on the information available in the General Agricultural and Livestock Census corresponding to the 2010/2011 production year. It was applied a stochastic boundary analysis to obtain a measure of the technical efficiency, as well as of each establishment. The results showed that Uruguayan dairy farmers operate at $79.9 \%$ of their capacity, which shows that many producers could still improve their incomes with the available technology. In addition, the results show that the technical assistance as well as the use of silos reduces the inefficiencies possible inefficiencies.
\end{abstract}

Keywords: technical efficiency; stochastic frontiers; milk production.

\section{Introdução}

A atividade de produção de leite ocupa um papel importante na economia do Uruguai. Em 2005, representou 9,7\% do produto interno bruto agropecuário, sendo o terceiro produto agropecuário mais importante depois da carne e da soja, que possuíam parcelas de $30 \%$ e 16\%, respectivamente. Em 2015, a produção total de leite foi de 2,141 milhões de litros, tendo sido 93\% processados. Existiam 3.919 estabelecimentos leiteiros, com 73,4\% deles vendendo o leite para a indústria de processamento. A área total utilizada para a produção leiteira correspondeu a 771 mil hectares (Dirección de Investigaciones Estadísticas Agropecuarias, 2017).

A maior parte da produção de leite é orientada para as exportações (70\%) na forma de leite em pó (em sua maioria) e produtos processados. As exportações de lácteos atingiram $8,1 \%$ do total de bens uruguaios exportados em 2015. Além disso, a produção leiteira é uma importante fonte de emprego. Segundo o Censo Agropecuário de 2011, o número de trabalhadores permanentes na pecuária leiteira representou $12,8 \%$ do total de trabalhadores permanentes no setor agrícola. A pecuária leiteira é o segundo setor em contratação de mão de obra após a produção pecuária, que contrata $48,4 \%$ do total de trabalhadores permanentes. 
Desde meados dos anos 1970, o setor de lácteos tem mostrado crescimento contínuo. Sua estrutura produtiva experimentou importantes mudanças, tendo gerado um notável dinamismo no setor. Esse dinamismo é refletido no maior volume de leite produzido e comercializado. Em 1976, a produção de leite era de 742 milhões de litros, tendo 47\% dela sido comercializada (347 milhões de litros) (Hernández, 2002). Em 2000, a produção de leite aumentou em $72 \%$, atingindo 1,278 milhão de litros. Além disso, o leite comercializado aumentou mais de três vezes, representando $82 \%$ do leite total produzido $(1,047$ milhão de litros). Essa tendência crescente, tanto de produção de leite quanto de leite comercializado, continuou durante os últimos anos, e a quantidade de leite comercializado quase dobrou entre 2000 e 2015 , tendo representado $93 \%$ do leite produzido.

O sistema de produção pastoril extensivo, baseado em pastagens naturais, evoluiu para um sistema intensivo de agricultura baseado em pastagens cultivadas e no uso de alimentos concentrados. Durán (2004) define cinco etapas para explicar a intensificação do sistema de produção de leite (as mais relevantes: produção e uso de pastagens cultivadas, uso de concentrados alimentícios e dotação de vacas por unidade de superfície), indicando que o nível de inovação tecnológica tem sido diferente entre os pecuaristas de leite. Porém, esse processo de mudança implicou um aumento contínuo no processo de incorporação da nova tecnologia, o que possibilitou a generalização de um sistema de produção intensivo. Tal fato permite assumir que as diferenças entre os produtores surgem de características tanto observáveis como inobserváveis que afetam o desempenho dos produtores no uso de uma mesma tecnologia e não no tipo de tecnologia utilizada.

As principais diferenças entre esses modelos são o uso de pastagens cultivadas, em vez de pastagens naturais, e maior oferta de alimentos concentrados e silagem. Essas mudanças na dieta das vacas levaram a melhorias no desempenho e a quantidade de vacas leiteiras por hectare aumentou. Segundo a Dirección de Investigaciones Estadísticas Agropecuarias (2017), a produtividade por vaca leiteira foi de 1.715 litros por ano em 1985, com uma taxa de crescimento anual entre 1985 e 2015 de 3,4\%. Além disso, durante o período 2000-2015, os agricultores alcançaram melhorias na produtividade. No entanto, o total de terras utilizadas para as explorações leiteiras diminuiu, o que mostra que a extensão de terra não foi um fator relevante para explicar o crescimento da produção de leite.

Consequentemente, os agricultores alcançaram um melhor desempenho pela intensificação do sistema de produção de leite, em termos de quantidades produzidas. Novas tecnologias e uso mais intensivo de recursos explicam o crescimento da produção. Por outro lado, a adoção de uma nova tecnologia implicou menores custos de produção, o que levou a um aumento da competitividade internacional do setor.

Embora a produção leiteira uruguaia tenha melhorado seu desempenho em termos de produção, todo o setor enfrenta importantes desafios para manter a competitividade no mercado internacional de lácteos. Trata-se de um dos mercados mais protegidos e subsidiados, sendo dominado por algumas empresas de grande porte que usam tecnologia moderna (Chaddad, 2009). Neste contexto, o crescimento da produtividade parece ser um mecanismo importante para melhorar a competitividade. Tanto as inovações tecnológicas quanto a eficiência com que a tecnologia disponível é utilizada influenciam o crescimento da produção.

O crescimento da produtividade pode ser definido por três componentes: eficiência técnica, mudança tecnológica e escala ou mudança de eficiência de tamanho (Coelli et al., 1985). O componente de produtividade mais estudado é a eficiência técnica, já que fornece informações valiosas para a formulação de políticas e decisões agrícolas focadas nas melhorias do desempenho da propriedade (Bravo-Ureta et al., 2008). Quando os estudos de desempenho de produtividade consideram os países em desenvolvimento, a análise da eficiência técnica é particularmente relevante, visto que nesses países há mais oportunidades para melhorar a prática gerencial, aprender fazendo e disseminar novas tecnologias, em comparação com as economias desenvolvidas. Portanto, em países em desenvolvimento, como o Uruguai, onde o processo de adoção de tecnologia pode enfrentar mais restrições, o estudo da eficiência técnica pode gerar informações valiosas sobre a capacidade dos agricultores de obter o máximo resultado dessa adoção, dado um 
conjunto de insumos e tecnologia. Existem oportunidades para melhorar o desempenho das explorações leiteiras, utilizando os recursos de forma mais eficiente sob a tecnologia de produção atual.

O objetivo deste estudo é estimar a eficiência técnica e analisar o desempenho dos produtores de leite no Uruguai, assim como as características que explicam as diferenças entre eles, segundo uma análise de fronteira de produção estocástica.

Apesar de se aplicar no trabalho, uma metodologia muito explorada internacionalmente, no caso do Uruguai, e especificamente no que tem a ver com economia agrícola, os estudos sobre eficiência baseados em fronteiras de produção são escassos. Por tal motivo, o presente estudo pretende dialogar com a literatura econômica referente à eficiência técnica da produção agrícola e aportar à literatura econômica nacional, dando elementos que permitam contribuir com o desenvolvimento do setor e das políticas que o envolvem.

Por tais motivos, espera-se fazer uma contribuição importante ao conhecimento econômico e, especialmente, do setor leiteiro no Uruguai, por ser a primeira análise de eficiência técnica da produção em âmbito nacional, levando em consideração todos os estabelecimentos leiteiros do país com base no censo agropecuário. Isso é especialmente relevante por ser um setor cuja realidade dos produtores apresenta, às vezes, grandes diferenças, como foi explicado anteriormente. Por isso, assume-se que poderá ser um insumo interessante para orientar as políticas de apoio e desenvolvimento do setor de forma mais diferenciada.

Cabe destacar também que o trabalho pretende contribuir no entendimento da realidade de um setor produtivo com características muito específicas, como foi detalhado antes. A maioria dos estabelecimentos se enquadra na classificação oficial de produtor familiar, por ser um setor com forte perfil exportador, e $90 \%$ dos produtores comercializam sua produção com a indústria (Dirección de Investigaciones Estadísticas Agropecuarias, 2017).

$\mathrm{O}$ artigo está organizado em quatro seções, além da introdução. A seguir, apresenta-se uma breve revisão da literatura sobre eficiência na produção de leite. A seção seguinte trata da metodologia empregada no estudo. Posteriormente, os resultados são apresentados e discutidos. Finalmente, apresentam-se as conclusões.

\section{Análise de eficiência na produção de leite}

As análises de eficiência baseadas em fronteiras têm uma ampla literatura. O método de programação matemática (não paramétrica) conhecida como Análises Envoltória de Dados (DEA) é um dos principais enfoques desenvolvidos para medir a eficiência produtiva. O segundo enfoque são as análises de fronteiras estocásticas (SFA), que é um método paramétrico que requer assumir uma forma funcional previamente definida.

Watkins et al. (2014) fazem referência a 20 trabalhos publicados entre 1991 e 2011, tendo a maioria deles utilizado a metodologia de SFA e somente oito aplicado o método DEA. As comparações feitas entre ambos os modelos não permitem concluir sobre a superioridade absoluta de um deles, porém Coelli et al. (1985) indicam que SFA tem sido a metodologia mais adotada para medir a eficiência da produção agropecuária por sua capacidade de considerar efeitos inobserváveis.

O anteriormente exposto motivou e justificou a escolha pela SFA para estimar a eficiência neste trabalho. Para isso, utilizaram-se os dados do Censo Geral Agropecuário de 2011, realizado pelo Ministerio de Agricultura Ganadería y Pesca del Uruguay (MEGAP), que correspondem ao exercício produtivo 2010/2011. A base tem uma vantagem fundamental: provê informações de todo o universo da pecuária leiteira do país. Porém, trata-se de uma base de corte transversal que não permite avaliar a evolução da eficiência nem o câmbio tecnológico. Outra limitação se refere às variáveis que determinam a fronteira por ter problemas de medição que dificultam a estima.

$\mathrm{Na}$ literatura internacional, o trabalho de Wang (2001) constitui a principal referência. Trata-se de uma análise de eficiência com base em uma amostra de 70 estabelecimentos leiteiros na Pensilvânia e Vermont, selecionados aleatoriamente, cujas informações correspondem a 1996. Estimaram-se uma fronteira de produção estocástica para dados de corte transversais, a eficiência média e a eficiência de cada um dos estabelecimentos. $\mathrm{O}$ 
autor assume três distribuições distintas do termo de ineficiência: seminormal, normal truncada e exponencial. A especificação da fronteira toma como variável dependente o total de leite produzida no ano (as livras de leite produzido por vaca multiplicado pelo número de vacas leiteiras) e como variáveis explicativas as horas de trabalho totais (familiares e remuneradas); o valor líquido médio dos ativos de capital e a alimentação (total de consumo de grãos e forragem por vaca multiplicado pela quantidade de vacas do estabelecimento). Além disso, a medida da eficiência técnica se subdivide por tamanho da exploração, tomando como aproximação o número de vacas leiteiras ${ }^{1}$, introduzido na fronteira por meio de duas variáveis dummies de controle. O autor conclui que, embora a eficiência técnica média tenha sido $85 \%$, os estabelecimentos maiores resultaram mais eficientes que os pequenos. Além disso, todos os estabelecimentos analisados mostraram rendimentos decrescentes na escala.

Cabrera et al. (2010) e Al-Sharafat (2013) estimaram a eficiência técnica usando uma fronteira de produção estocástica baseada em uma amostra transversal de produtores de leite. Cabrera et al. (2010) analisaram os efeitos das práticas comumente usadas pelos produtores de leite e o efeito da intensificação no desempenho das fazendas. Uma amostra de 273 agricultores em Wisconsin foi utilizada para estimar a fronteira estocástica e o modelo de ineficiência técnica. Os resultados empíricos mostraram que o nível médio de eficiência técnica (ET) na amostra foi de $88 \%$, indicando que os agricultores poderiam expandir a produção de leite usando os insumos e a tecnologia disponível. A variável com maior efeito sobre a produção foi o número de vacas seguida pelo gasto total com lavouras, alimentação, pecuária e trabalho. Uma relação proporcional entre o tamanho da fazenda e o nível de ET não foi encontrada, o que sugere que melhorias na tecnologia e na eficiência explicam o nível de produtividade, mas não o tamanho da fazenda.

Al-Sharafat (2013) utilizou uma metodologia de fronteira de produção estocástica para obter o nível de ET de fazendas leiteiras na Jordânia. O estudo encontrou um baixo nível de ET para a maioria das 12 fazendas leiteiras, tendo a ET média na amostra de 100 fazendas sido de $39,5 \%$. O tamanho da manada, o consumo de ração e os custos com mão de obra e serviços veterinários tiveram um efeito positivo e significativo na produção de leite. No entanto, o tamanho do rebanho foi a variável explicativa mais importante. Os principais determinantes da ET que tiveram um efeito positivo foram: o nível de escolaridade do agricultor, a experiência agrícola do agricultor, o contato do agricultor com os serviços de extensão e o tamanho do rebanho. O autor concluiu que mais educação é fundamental para alcançar um melhor desempenho de eficiência entre os produtores de leite na Jordânia.

Finalmente, Mbaga et al. (2003) estimaram o nível de ET de fazendas leiteiras em Quebec usando uma seção transversal de 1.143 fazendas. Eles mediram e avaliaram a robustez da ET escolhendo diferentes formas funcionais para a fronteira e diferentes pressupostos de distribuição para o termo de ineficiência. A abordagem de análise de fronteira estocástica foi utilizada e, para fins de comparação, a análise por envoltória de dados (DEA) também foi utilizada para estimar a ET. Altos níveis de eficiência foram obtidos para fazendas de gado leiteiro em Quebec. Além disso, os resultados mostram que as diferenças nos níveis médios de eficiência foram estatisticamente significativas entre as formas funcionais e as distribuições dos termos de ineficiência. No entanto, as diferenças nas magnitudes médias de ET não foram muito grandes.

Nascimento et al. (2012) analisaram a eficiência na produção leiteira em Minas Gerais, Brasil, segundo os efeitos de variáveis técnicas e econômicas sobre a fronteira de produção estocástica, com base em uma regressão quantílica. Foram utilizadas informações referentes a 875 produtores do estado, coletadas em 2005. Do estudo se deduz que ainda há uso extensivo do fator terra e que os produtores poderiam reduzir a área utilizada para a produção de leite sem perda de eficiência, sendo a proporção de vacas lactantes um fator relevante para explicar o grau de eficiência técnica em todos os quantis. Além disso, o trabalho familiar só se mostra significativo para explicar o nível de eficiência naqueles

\footnotetext{
1 Vinte e quatro estabelecimentos foram classificados como pequenos por terem menos de 50 vacas leiteiras; 30, medianos,
} por possuírem entre 50 e 100 vacas leiteiras e 16 grandes explorações, por terem mais de 100 vacas leiteiras. 
produtores menos eficientes. Por último, para efeitos comparativos, verificou-se que a eficiência técnica média dos produtores foi de $82,6 \%$.

No trabalho mais recente de Silva \& Bragagnolo (2018), estimou-se uma função de produção, por meio de análise econométrica de fronteira estocástica, e avaliou-se a eficiência técnica e ambiental na produção de leite na região do Triângulo Mineiro e Alto Paranaíba, com informações de 178 produtores de leite dessas regiões, em 2016. A análise apontou que a média da ET obtida pelos estabelecimentos analisados foi de $89 \%$, enquanto a da eficiência ambiental foi de 73\%, havendo uma correlação positiva entre ambas.

Guerrero et al. (2017) analisaram a ET na produção leiteira do Pampa argentino (Buenos Aires, Córdoba e Santa Fé), com base em um painel de dados de 86 estabelecimentos em dois períodos produtivos (julho de 2012 a junho de 2013 e julho de 2014 a junho de 2015). Estimouse a ET por meio de fronteiras estocásticas de produção, estabelecendo controles pela heterogeneidade observada e pela não observada. Os resultados mostraram que houve pequena redução da eficiência técnica média, que passou de $88,1 \%$ a $87,8 \%$ em termos gerais, tendo sido a região de Buenos Aires a única a apresentar incremento no grau de eficiência (de $85,4 \%$ a $90,5 \%)$.

Os trabalhos de Grau et al. (1995) e Bravo-Ureta et al. (2008) analisaram a eficiência da produção leiteira no Uruguai. Grau et al. (1995) verificaram o grupo de estabelecimentos leiteiros que integram os grupos CREA (Centros Regionais de Experimentação Agropecuária). Os autores estimaram a fronteira de produção determinística e avaliaram, com base nela, a eficiência dos estabelecimentos no uso da tecnologia disponível. Os autores consideraram 537 registros entre 1978 e 1991 contendo informações referentes ao tamanho dos estabelecimentos, à superfície e à intensidade das pastagens plantadas, de ração, à carga animal e aos níveis dos resultados econômicos obtidos (estimados em termos de produtividade por unidade de superfície). Os resultados obtidos confirmaram a hipótese do potencial em termos de produtividade que teria intensificação da alimentação forrageira. Em termos gerais, constataram-se altos níveis de eficiência nos estabelecimentos considerados, sendo pouco provável que possam atingir maiores níveis de produtividade e rentabilidade sem modificar a tecnologia. Só com um movimento de fronteira tecnológica poder-se-ia alcançar níveis de produção potencialmente maiores.

Mais recentemente, Bravo-Ureta et al. (2008) analisaram as economias de escala, taxas de câmbio tecnológico e eficiência técnica, mediante estimativa das fronteiras estocásticas de produção, aplicadas a uma amostra de estabelecimentos leiteiros do Uruguai, Argentina e Chile, nos períodos de 1999 a 2003, 1997 a 2002 e 1996 a 2002, respectivamente. Trabalhou-se com 46 prédios situados na província de Buenos Aires, 48 estabelecimentos do sul do Chile e 70 no sul do Uruguai. As variáveis consideradas foram a produção promédio anual de leite (medida em litros), o número promédio de vacas por prédio leiteiro, a mão de obra (trabalhadores equivalentes), o gasto com alimentos (concentrados, forragem, minerais e custos associados) e com insumos veterinários. Além disso, define-se uma variável de tendência que captura o câmbio tecnológico e modifica-se com cada conjunto de dados. As três fronteiras foram calculadas utilizando a especificação translogarítmica. O resultado das estimativas para os três países mostrou economias de escala crescentes, concluindo-se que os estabelecimentos analisados têm trabalhado em tamanho subótimo. Por sua vez, encontrou-se uma taxa promédio anual de câmbio tecnológico de 0,9\% para a Argentina, 2,6\% pra o Chile e 6,9\% para o Uruguai. A eficiência técnica média estimada foi de $87 \%, 84 \%$ e $81,1 \%$, respectivamente.

\section{Metodologia}

A metodologia de fronteira de produção como forma de medir a eficiência foi introduzida por Farrell (1957), sendo a ideia base a comparação dos resultados produtivos de cada unidade quanto à melhor prática. O modelo de fronteira proposto por Farrell é conhecido como determinístico, em que qualquer desvio de fronteira é atribuído à ineficiência, e não paramétrico, já que não é considerada a priori nenhuma forma funcional.

A metodologia de fronteiras tem contado com muitas contribuições desde o trabalho pioneiro. Uma das mais relevantes tem sido o desenvolvimento das fronteiras de produção 
estocásticas, cuja especificação inclui simultaneamente uma perturbação aleatória e um término de ineficiência. Os trabalhos desenvolvidos de forma independente por Aigner et al. (1977) e Meeusen \& Van den Broeck (1977) reconhecem a existência de elementos inobserváveis, que geram ruído na estimativa, mas não podem ser incluídos na fronteira, resolvendo, assim, o principal problema do enfoque determinístico, que é imputar como ineficiência qualquer desvio da fronteira, o que, na produção agropecuária, significaria, por exemplo, omitir o efeito que as condições ambientais e as climáticas têm sobre os rendimentos produtivos.

Nesse sentido, o enfoque seguido neste trabalho para estimar a eficiência corresponde à análise de fronteiras estocásticas para dados de corte transversais. Como fora explicado, trata-se de um método paramétrico que considera que as firmas não têm controle sobre a totalidade dos fatores que afetam o processo produtivo. Assim, para uma combinação de insumos, existe um produto ótimo e possível que é aleatório e não exato. Supõe-se conhecida a estrutura do conjunto de possibilidades de produção e do processo gerador de dados (assume-se uma distribuição para o resíduo no modelo) para um conjunto de parâmetros desconhecidos.

Segundo o trabalho de Bogetoft \& Otto (2011), a representação geral da fronteira estocástica pode-se escrever como:

$Y_{i}=\exp \left(x_{i} \beta+\varepsilon_{i}\right)$

$\varepsilon_{i}=v_{i}-u_{i}$

Onde $Y_{i}$ representa a produção dos $i$ estabelecimentos da amostra, $x_{i}$, o vetor $(1 x K)$ de fatores de produção (insumos e outras variáveis explicativas), $\beta$, o vetor (KxI) dos parâmetros desconhecidos a estimar associados às variáveis independentes, e o produto vetorial corresponde à função de produção ${ }^{2}$. Por último, $\varepsilon_{i}$ corresponde ao termo de perturbação composto de uma variável aleatória irrestrita $\left(v_{i}\right)$ e pelo termo de ineficiência $\left(\mathrm{u}_{\mathrm{i}}\right)$, que é não positivo, ambos independentes entre si. Enquanto $v_{i}$ considera-se independente e identicamente distribuído como $N\left(0, \sigma_{v}^{2}\right)$, o termo captura a ineficiência $u_{i}$, supõe-se geralmente independente e identicamente distribuído como uma normal truncada $N^{+}\left(\mu, \sigma_{u}^{2}\right)$ com média diferente de zero. Isso significa que se um produtor fosse ineficiente, o valor de $u_{i}$ teria que ser maior do que zero.

Coelli et al. (1985) sinalaram que na literatura há diferentes propostas para a distribuição do termo de ineficiência: seminormal (Aigner et al., 1977), normal truncada (Stevenson, 1980), gama (Greene, 1980) e exponencial (Meeusen \& Van den Broeck, 1977). Porém, quando se escolhe uma distribuição seminormal ou exponencial, a maioria dos efeitos da ineficiência ficou no entorno de zero e as medidas associadas à eficiência técnica situaram-se no entorno de um, o que não acontece quando o modelo adota uma distribuição normal truncada ou gama que permitem maior variabilidade.

Estima-se o modelo pelo método de máxima verossimilhança (MV) cujas propriedades assintóticas permitem superar os vieses das estimativas do mínimo quadrado ordinário (MCO). As condições de primeira ordem são não lineares, obtendo-se o valor dos parâmetros de interesse para estimar a eficiência segundo um procedimento de otimização iterativa.

Ao assumir a forma normal truncada, a distribuição do termo de ineficiência se expressa como:

$$
f(u)=\left(1 / \sigma_{u}\right) \phi\left[(u-\mu) / \sigma_{u}\right] / \Phi\left[\mu / \sigma_{u}\right]
$$

${ }^{2} \mathrm{~A}$ forma funcional mais comum é do tipo Cobb-Douglas, mas a translogarítmica tem a vantagem de ser mais flexível e uma generalização da primeira. 


$$
\begin{aligned}
& E(u)=\mu+\frac{\sigma_{u} \phi\left[\mu / \sigma_{u}\right]}{\phi\left[\mu / \sigma_{u}\right]}=\mu+\sigma_{u} \lambda_{u} \\
& \operatorname{Var}[u]=\sigma_{u}^{2}\left[1-\lambda_{u}\left(\mu / \sigma_{u}+\lambda_{u}\right)\right]
\end{aligned}
$$

Com a parametrização proposta por Aigner et al. (1977) e a função de máxima verossimilhança (FMV) expressada em função de $\sigma^{2}=\sigma_{v}^{2}-\sigma_{u}^{2}$, a importância do termo de ineficiência pode se representar como $\lambda^{2}=\sigma_{u}^{2} / \sigma_{v}^{2} \geq 0$. Alternativamente, Battese \& Corra (1977) propõem o parâmetro $\gamma=\sigma_{u}^{2} / \sigma^{2}$ como indicador da relevância do termo de ineficiência no modelo que toma valores entre zero e um. No caso em que $\gamma=0$, os desvios da fronteira explicam-se completamente pelo erro estocástico, sendo nulos os efeitos da ineficiência técnica, e o resultado seria o mesmo que na estimativa por $\mathrm{MQO}^{3}$.

A estimativa da eficiência técnica para cada estabelecimento resulta da seguinte forma:

$$
E T_{i}=\widehat{E T}=e^{-E V(u / \hat{\varepsilon})}=\exp \left(-\hat{u}_{i}\right)
$$

Assim, a eficiência técnica depende só da estimativa do termo de ineficiência. Porém, deve-se estimar primeiro o termo de erro conjunto e, depois, estabelecer a distribuição condicional de $u_{i}$, dado $\varepsilon_{i}$. Assim se obtém uma estimativa consistente do termo de ineficiência cuja forma é a seguinte: $\widehat{u_{i}}=E V(u / \varepsilon)=\mu_{*}+\sigma_{*} \frac{\phi\left(\mu_{*} / \sigma_{*}\right)}{\Phi\left(\mu_{*} / \sigma_{*}\right)}$

Onde $\mu_{*}=-\varepsilon \frac{\sigma_{u}^{2}}{\sigma^{2}}=-\varepsilon \frac{\lambda^{2}}{1+\lambda^{2}}=-\varepsilon \gamma \sigma_{*}=\sqrt{\frac{\sigma_{u}^{2} \sigma_{v}^{2}}{\sigma^{2}}}=\frac{\lambda}{1+\lambda^{2}} \sigma=\sqrt{\gamma(1-\gamma) \sigma^{2}}$

Define-se, então, o estimador da eficiência técnica como: $\widehat{E T}=e^{-E V(u / \hat{\varepsilon})}=e^{-\hat{u}}$, com base nas estimativas de $\varepsilon, \sigma^{2} e \gamma$, que são os parâmetros estimados diretamente no modelo.

O estimador ótimo que minimiza o erro quadrado médio usualmente utilizado resulta como:

$$
E T=E V\left(e^{-u} \mid \varepsilon\right)=\frac{\Phi\left(\mu_{*} / \sigma_{*}-\sigma_{*}\right)}{\Phi\left(\mu_{*} / \sigma_{*}\right)} e^{\left(\frac{1}{2} \sigma_{*}^{2}-\mu_{*}\right)}
$$

Sendo a eficiência média para o total da amostra: $\overline{E T}=\frac{1}{I} \sum_{i=1}^{I} \widehat{E T}{ }_{i}$

Segundo o modelo geral, pode-se obter a medida da eficiência técnica promédio e de cada estabelecimento. Como foi dito, os efeitos da ineficiência materializados como desvios relacionados à fronteira associam-se a fatores aleatórios fora do controle do produtor.

${ }^{3} \mathrm{O}$ teste de significação estadística dos efeitos da ineficiência corresponde ao contraste da hipótese nula H0: y = 0 contra H1: $y>0$. Toda vez que o LR (estadístico da razão de verossimilhança) excede o valor crítico de $\chi_{-}(1-2 a)^{\wedge} 2$ (1), a hipótese nula é rejeitada.

${ }^{4} \mathrm{~A}$ densidade do termo do erro $\varnothing_{u}(u)=\frac{1}{\sqrt{2 \pi \sigma_{v}^{2}}} e^{-\frac{1}{2} \frac{v^{2}}{\sigma_{v}^{2}}}$ corresponde a la distribución normal estándar. Mientras que la distribución del término de ineficiencia $\varnothing_{u}(u)=\left\{\begin{array}{cc}\frac{2}{\sqrt{2 \pi \sigma_{u}^{2}}} e^{-\frac{1}{2} \frac{u^{2}}{\sigma_{u}^{2}}} & \text { parau } \geq 0 \\ 0 & \text { para } u<0\end{array}\right.$.

Y Por último, la densidad del término conjunto $\varnothing_{\varepsilon}(\varepsilon)=\frac{\sqrt{2}}{\sqrt{\pi \sigma^{2}}} \Phi\left(-\frac{\lambda \varepsilon}{\sqrt{\sigma^{2}}}\right) e^{-\frac{1}{2} \frac{\varepsilon^{2}}{\sigma^{2}}}$, siendo $\sigma^{2}=\sigma_{v}^{2}+\sigma_{u}^{2}, \lambda=\sqrt{\frac{\sigma_{u}^{2}}{\sigma_{v}^{2}}}$ y $\Phi(x)$ la distribución normal estándar. 
Porém, é possível assumir que grande parte das possíveis ineficiências no processo produtivo seja consequência das decisões do produtor. Uma modelização alternativa do termo de ineficiência poderia introduzir outros fatores observáveis na fronteira que teriam efeito no resultado produtivo, como são as condições ambientais, as condições sociais do produtor ou as decisões de adoção tecnológica, entre outras.

Battese \& Coelli (1995) propuseram um modelo mais geral do que outros autores, como Kumbhakar et al. (1991) tinham assumido, especificando o termo de ineficiência como uma função das características observáveis mencionadas no parágrafo anterior:

$u_{i}=g\left(z_{i}\right)+\varepsilon_{i}$

Onde $\varepsilon_{i} \sim N^{+}\left(0, \sigma_{u}^{2}\right)$ y g(.) é uma função não negativa, tal que $u_{i} \sim N^{+}\left(z_{i}^{\prime} \delta, \sigma_{u}^{2}\right)$.

Assim, a estrutura convencional da fronteira estocástica normal-truncada na versão aditiva resulta como:

$\ln y_{i}=x_{i}^{\prime} \beta-g\left(z_{i}\right)+v_{i}-\varepsilon_{i}$

Sem modificar o processo de estimativa do modelo e da eficiência técnica, que segue sendo o assinalado anteriormente.

\section{Variáveis, fontes de dados e tratamento empírico}

Ao implementar uma abordagem paramétrica, é necessária uma forma funcional específica para a fronteira de produção. Utiliza-se um teste de razão de verossimilhança ${ }^{5}$ para ajudar a confirmar qual forma funcional se ajusta significativamente melhor aos dados. Inicialmente se testou se uma função translogarítmica se ajusta melhor aos dados que a Cobb-Douglas, que é uma forma mais restrita. Os resultados mostram que não é possível rejeitar a hipótese nula $\left(H_{0}: \beta_{i j}=0\right)$ de que a forma funcional aninhada pode ser justificada com nível de significância de $5 \%$, o que significa que a forma Cobb-Douglas é uma especificação mais adequada a nossos dados $(\lambda=14,47 ; p>0,05)$. Assim, o modelo empírico deste estudo baseia-se na estimativa de uma função de produção estocástica do tipo CobbDouglas (o que foi escolhido por parcimônia, atendendo à simplicidade da especificação e da análise com respeito à forma translogarítmica como já foi explicado). As variáveis explicativas são expressas em forma logarítmica da seguinte forma:

$$
\begin{aligned}
& \ln Y_{i}=\beta_{0}+\beta_{1} \ln x_{1 i}+\beta_{2} \ln x_{2 i}+\beta_{3} \ln x_{3 i}+\beta_{4} \ln x_{4 i}+v_{i}-u_{i} \\
& u_{i}=\delta_{1} z_{1 i}+\delta_{2} z_{2 i}+\delta_{3} z_{3 i}+\delta_{4} z_{4 i}+w_{i}
\end{aligned}
$$

Onde os subíndices se referem aos i estabelecimentos da amostra e tomando as variáveis em logaritmos. Do lado direito, a produção de leite anual medida em litros é a variável dependente. Do lado esquerdo: $x_{i}$ (vacas) representa a soma do número de vacas em ordenha e o número de vacas secas; $x_{2}$ (pastagens) mede-se como a superfície de pastagens melhoradas ou não dedicada ao gado de leite; $x_{3}$ (trabalho) representa a quantidade total de horas de trabalho remunerado (foi calculada multiplicando o número de trabalhadores remunerados permanentes declarados pelos produtores vezes a jornada anual promédio por trabalhador de 2.100 horas); $\mathrm{x}_{4}$ (maquinaria) corresponde ao valor da

\footnotetext{
${ }^{5} \mathrm{~A}$ estatística do teste da razão de verossimilhança é calculada como: $\lambda=-2\left[\log \left(\operatorname{likelihood}\left(H_{0}\right)-\log \left(\operatorname{likelihood}\left(H_{0}\right.\right.\right.\right.$ )] e possui distribuição $\chi^{2}$ com parâmetro igual ao número de parâmetros assumidos como zero sob hipótese nula.
} 
maquinaria declarada pelo produtor (aplicou-se o preço promédio anual no ano do censo, mas não foi possível calcular a depreciação por falta de informações nos registros).

As variáveis que compõem o modelo de ineficiência são as seguintes: $z_{1}$ indica o uso de serviços veterinários e assume valor 1 quando realizados inseminações artificiais ou diagnósticos gestacionais, e 0 , caso contrário; $z_{2}$ indica a utilização de assistência técnica agronômica ou contável: vale 1 quando o produtor declarou ter usado esses serviços e 0 caso contrário; $z_{3}$ indica o nível educativo, assumindo valor 1 quando o produtor finalizou 0 ensino médio e 0 caso contrário; $z_{4}$ vale 1 se o produtor declarou utilizar silos para armazenar grãos e 0 caso contrário.

Para as estimativas, utilizou-se o modelo de máxima verossimilhança para estimar os parâmetros da fronteira estocástica, supondo-se uma distribuição normal-truncada do termo de erro $\left(\varepsilon_{\mathrm{i}}\right)$.

Os dados foram coletados no Censo Geral Agropecuário (CGA) correspondente ao exercício produtivo de 2010/2011 cujo relevamento é de caráter nacional e realiza-se cada dez anos pela Direção de Estatísticas Agropecuárias (Diea) do Ministério de Pecuária Agricultura e Pesca do Uruguai (MGAP).

Da informação registrada, depreende-se que um total de 4.474 estabelecimentos realizou leiteria em âmbito comercial nesse ano, ocupando uma superfície de pouco mais de um milhão de hectares, com 744.909 cabeças de gado leiteiro que permitiram produzir um total de 1.780.146.198 litros de leite. Para a presente análise, consideram-se só os estabelecimentos que têm remitido a produção para a indústria, o que significa um total de 3.201 produtores, o que representa $94,4 \%$ da produção total.

As variáveis analisadas que definem a fronteira são aquelas relevante na produção leiteira de acordo com a literatura na matéria. De acordo com isso, realizou-se uma análise de correlação das variáveis disponíveis nos registros procurando as melhores aproximações dos determinantes da produção leiteira: para trabalho, alimentação, equipamento e rodeio leiteiro (número de cabeças de gado leiteiro).

A Tabela 1 apresenta as estatísticas descritivas das respectivas variáveis empregadas no estudo

Tabela 1. Análise descritiva das variáveis

\begin{tabular}{cccc} 
Variáveis & Nome & Média & Erro padrão \\
Produção (l) & $\mathrm{Y}_{\mathrm{i}}$ & 517658,8 & 944388,2 \\
Vacas (número de cabeças) & $\mathrm{x}_{1}$ & 229 & 106,2 \\
Pastagens (ha) & $\mathrm{x}_{2}$ & 224 & 230 \\
Trabalho remunerado (h) & $\mathrm{x}_{3}$ & 6044,8 & 13983,1 \\
Maquinaria (dólares) & $\mathrm{x}_{4}$ & 83253,7 & 45443,1 \\
\hline
\end{tabular}

Fonte: resultados da pesquisa.

\section{Resultados}

De acordo com o teste de razão de verossimilhança ${ }^{6}$, comparou-se o ajuste do modelo na forma Cobb-Douglas e na forma translogarítmica, o que resultou na especificação CobbDouglas mais adequada ao nível de significação de $5 \%$, pelo teste de razão de máxima verossimilhança (LR teste). Assume-se, então, que o modelo Coob-Douglas tem o melhor ajuste aos dados disponíveis pelo que todos os resultados a serem apresentados baseiamse nessa especificação do modelo.

No modelo estimado, todas as variáveis explicativas resultaram estatisticamente significativas (Tabela 2), revelando, assim, as elasticidades dos insumos, por se tratar de uma função de produção Cobb-Douglas na forma logarítmica. A estimativa indica que o incremento percentual unitário da quantidade de vacas no rodeio, a superfície de pradarias,

${ }^{6}$ O estatístico LR-teste compara por diferença dos modelos, assumindo uma distribuição $\chi^{2}$ baixa a hipótese nula, sendo rejeitada para um p-valor muito pequeno. 
o trabalho e a maquinaria têm como resultados um incremento na produção de leite de 0,793\%, 0,194\%, 0,082\% e 0,091\%, respectivamente.

Pode-se observar também que os coeficientes das variáveis que definem a fronteira de produção (vacas, superfície de pastagens, trabalho e maquinaria) resultaram de sino não negativo como era de se esperar, o que significa que todas elas influem positivamente sobre a quantidade de leite produzida, satisfazendo a propriedade de monotonocidade.

Tabela 2. Parâmetros estimados: fronteira de produção

\begin{tabular}{ccc} 
Variável & Coeficiente estimado & Desvio padrão \\
Constante & $6,446^{* *}$ & 0,203 \\
Vaca & $0,793^{* * *}$ & 0,015 \\
Pradarias & $0,194^{* * *}$ & 0,016 \\
Trabalho & $0,082^{* *}$ & 0,012 \\
Maquinaria & $0,091 * *$ & 0,019 \\
Constante & $0,923^{* * *}$ & 0,097 \\
Assistência técnica & $-0,098^{* *}$ & 0,023 \\
Silos & $-0,247^{* *}$ & 0,091 \\
Assistência veterinária & $-0,336^{* * *}$ & 0,101 \\
Educação & 0,013 & 0,002 \\
$\sigma^{2}$ & $0,176^{* * *}$ & 0,013 \\
$\gamma$ & $0,824 * * *$ & 0,074 \\
Log verossimilhança & 35,3 & \\
\hline
\end{tabular}

Código de significação do LR-teste: ***: 0,001; **: 0,01; *: 0,05-0,1. Fonte: resultados da pesquisa.

A soma dos coeficientes das variáveis mencionadas totaliza 1,16 , indicando que a tecnologia tem retornos crescentes à escala, o que significa que um incremento dado nos insumos teria como resultado um incremento maior na produção de leite, portanto a produtividade total dos fatores seria crescente. Confirmou-se o resultado anterior, com base no teste de razão de verossimilhança (LR-teste), sendo a hipótese nula a presença de retornos constantes à escala (soma dos coeficientes igual a um), rejeitando-se os $5 \%$ de significação. As economias de escala sugerem que, além das melhoras tecnológicas e da eficiência, o maior tamanho dos estabelecimentos também influi para alcançar mudanças na produtividade. Porém, além de as economias de escala poderem indicar uma alta estrutura de custos difícil de superar pelos pequenos produtores, é importante assinalar que as debilidades da base de dados (ser de corte transversal e ter problemas de medição nas variáveis) poderiam relativizar esse resultado, indicando o que Bravo-Ureta et al. (2008) encontraram no seu trabalho.

Com um valor de gama de 0,624, confirmam-se a relevância do termo de ineficiência e a adequação do modelo de fronteira estocástica (em relação a uma estimação MQO), testando-se a significação do parâmetro a partir do teste de razão de máxima verossimilhança. A hipótese de não significação do parâmetro foi rejeitada a $1 \%$ de significação.

No modelo de ineficiência, as variáveis introduzidas resultaram significativas sem ser educação; o sinal negativo indica que, ao manter todo o resto constante, os produtores que utilizaram assistência veterinária ou de outro tipo, ou realizaram ensilagem de grão, foram relativamente menos ineficientes, ou seja, capazes de alcançar maiores níveis de eficiência em relação àqueles que não adotaram essas técnicas. Assistência veterinária e realização de ensilagem resultaram nos determinantes que mais contribuíram para reduzir a ineficiência quando foram utilizados.

Em relação à eficiência técnica, o valor estimado foi de 0,797 , o que indica que, em média, os produtores leiteiros do Uruguai alcançaram praticamente $80 \%$ da capacidade 
produtiva. Um resultado bastante similar também foi apresentado no trabalho de BravoUreta et al. (2008) cujo valor estimado resultou em 0,811. Tal fato indica que os produtores ainda poderiam melhorar os resultados aplicando práticas produtivas mais eficientes, ou seja, os produtores leiteiros em promédio ainda têm $20 \%$ da capacidade tecnológica disponível para obter melhoras nos rendimentos.

A análise descritiva apresentado na Tabela 3 indica que 50\% dos estabelecimentos apresentaram valor de eficiência menor do que 0,804 , valor levemente maior que a média $(0,797)$. Além disso, o valor da eficiência máxima $(0,928)$ resultou levemente menor do que 1 , o que poderia indicar que os produtores mais eficientes não estariam alcançando o ótimo para a tecnologia disponível. Esses resultados condizem com os obtidos por Bravo Ureta et al. (2008), no que se refere à eficiência média, que foi de 0,8110 , porém, tendo eles analisado uma amostra restringida (só considerou produtores da região Sul do país), os valores extremos de eficiência foram 0,4930 e 0,971, mínimo e máximo, respectivamente.

Tabela 3. Resumo estatístico da eficiência estimada

\begin{tabular}{cccccc} 
Mínimo & $\mathbf{Q}_{\mathbf{1}}$ & Mediana & Media & $\mathbf{Q}_{\mathbf{3}}$ & Máximo \\
\hline 0,168 & 0,755 & 0,804 & 0,797 & 0,845 & 0,928 \\
\hline
\end{tabular}

Fonte: resultados da pesquisa.

Da análise de correlação entre a eficiência técnica e a quantidade de leite produzida, depreende-se que existe uma relação significativamente positiva entre ambas que alcança 0 valor de 0,623 . Esse resultado era de se esperar, dado o efeito direto que a eficiência tem sobre o volumem produzido, conforme mostra a Tabela 4. Enquanto a correlação da eficiência diz respeito às variáveis explicativas, observa-se uma relação mais frágil especialmente em relação ao trabalho e à maquinaria.

Tabela 4. Correlação entre eficiência e variáveis da fronteira

$\begin{array}{cccccc} & \text { Produção } & \text { Vacas } & \text { Pradarias } & \text { Trabalho } & \text { Maquinaria } \\ \text { Eficiência } & 0,623 & 0,491 & 0,366 & 0,023 & 0,125\end{array}$

Fonte: resultados da pesquisa.

Para analisar a significância das correlações, foi aplicado o teste de correlações de Pearson, que supõe uma correlação nula como hipótese do contraste, tendo resultado todas as correlações significativas a $1 \%$, com exceção da maquinaria, que só resultou significativa aos $5 \%$.

Para determinar um perfil que caracterizasse os estabelecimentos mais eficientes, utilizaram-se os quartis de eficiência que dividiram a amostra em quatro grupos e calculouse a média de cada variável explicativa. A Tabela 5 mostra o resumo do cálculo e o resultado do teste de médias aplicado, em que letras distintas indicam diferença estatisticamente significativa entre grupos para cada variável.

Tabela 5. Média de cada variável explicativa por faixa de eficiência

\begin{tabular}{lcccc|cc} 
Faixas & $\begin{array}{c}\text { Produtores } \\
(\mathbf{n})\end{array}$ & $\begin{array}{c}\text { Produção } \\
(\mathbf{I s})\end{array}$ & $\begin{array}{c}\text { Vacas } \\
(\mathbf{n})\end{array}$ & $\begin{array}{c}\text { Maquinaria } \\
\text { (dólares) }\end{array}$ & $\begin{array}{c}\text { Pastagens } \\
\text { (ha) }\end{array}$ & $\begin{array}{c}\text { Trabalho } \\
\text { (h) }\end{array}$ \\
\hline Faixa 1 & 797 & $152328^{\mathrm{a}}$ & $137^{\mathrm{a}}$ & $67711,2^{\mathrm{a}}$ & $98^{\mathrm{a}}$ & $2694^{\mathrm{a}}$ \\
Faixa 2 & 801 & $342588^{\mathrm{ab}}$ & $209^{\mathrm{ab}}$ & $78249,1^{\mathrm{a}}$ & $184^{\mathrm{a}}$ & $5118^{\mathrm{a}}$ \\
Faixa 3 & 825 & $706306^{\mathrm{b}}$ & $251^{\mathrm{ab}}$ & $86804,8^{\mathrm{a}}$ & $230^{\mathrm{ab}}$ & $6842^{\mathrm{b}}$ \\
Faixa 4 & 766 & $832275^{\mathrm{c}}$ & $309^{\mathrm{b}}$ & $89920,8^{\mathrm{a}}$ & $253^{\mathrm{b}}$ & $9087^{\mathrm{b}}$ \\
\hline
\end{tabular}

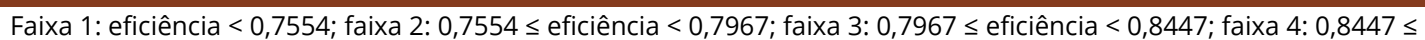
eficiência $\leq 1$. Fonte: resultados da pesquisa. 
A produção de leite resultou em média significativamente distinta entre os grupos de baixa e alta eficiências, o que confirma a correlação positiva entre eficiência e volume de leite produzido.

Com respeito às variáveis explicativas, o tamanho do rebanho leiteiro mostrou diferença significativa unicamente para as faixas extremas, ocorrendo o mesmo para a superfície de pastagens. Quanto ao fator maquinaria, permitiu estabelecer diferenças significativas entre os produtores que se encontram acima da média de eficiência e os que estão abaixo. Quanto ao trabalho, as diferenças não resultaram significativas, o que condiz com as correlações antes analisadas.

Assim, o resultado estaria indicando que os estabelecimentos mais eficientes são maiores em promédio no tamanho do rodeio, na superfície de pastagens e no uso de maquinaria.

\section{Conclusões}

O presente estudo estimou a eficiência técnica e analisou as características dos produtores de leite no Uruguai que explicam as diferenças de eficiência entre eles, com base em uma análise de fronteira de produção estocástica.

Os resultados obtidos na estimativa (em que a função de produção Cobb-Douglas demonstrou ser a mais adequada aos dados) indicaram a existência de retornos crescentes à escala, o que poderia indicar que, além das melhoras tecnológicas e de eficiência, o tamanho (a escala dos estabelecimentos) é um fator importante para alcançar melhorias na produtividade. Apesar das considerações sobre a estrutura de custos que é difícil de ser modificada pelos pequenos produtores e compromete suas melhorias, é importante assinalar que problemas na medição do trabalho e da maquinaria, assim como a falta de informações adequadas sobre custos de alimentação do rodeio, requerem relativizar o resultado. Para futuros trabalhos, seria pertinente realizar uma análise de eficiência de custos que permita avaliar mais profundamente esses aspectos.

Quanto aos fatores produtivos, o número de vacas, as pradarias, o valor da maquinaria e as horas trabalhadas resultaram significativos e com efeito positivo sobre o volume de leite produzido, sendo o número de vacas a variável com efeito preponderante. A eficiência média estimada foi dos $79,7 \%$, indicando que os produtores poderiam melhorar a capacidade produtiva em média, ser mais produtivos e eficientes, adequando melhor o uso da tecnologia, ou seja, é possível incrementar o volume de leite produzido em média, utilizando o nível atual de insumos.

Além disso, a utilização de assistência veterinária (para inseminação e diagnóstico gestacional) foi o principal determinante dos ganhos de eficiência, seguido do uso de ensilagem de grãos e assistência agronômica ou contável. O nível de educação não resultou uma variável significativa para explicar as possíveis ineficiências.

Por último, ao analisar o perfil dos produtores, observa-se que o grupo dos mais eficientes conseguiu níveis de produção médio maiores do que os do grupo dos menos eficientes. Além disso, o grupo mais eficiente é integrado por estabelecimentos maiores em termos do rodeio, com mais superfície de pastagens em relação àqueles menos eficientes. Isso indica que ainda subsiste uma forma de produção extensiva nos mais eficientes, portanto se poderia reduzir a superfície ocupada na produção leiteira sem perda do grau de eficiência.

Apesar das contribuições feitas por este trabalho, trata-se apenas de uma primeira aproximação que poderá ser aprimorada em futuros trabalhos. Ao considerar, principalmente, as debilidades da base de dados no que se refere à maquinaria específica (investimento em máquinas de ordenha, por exemplo), essa variável tem pouca relevância explicativa no modelo, e a falta de informações sobre alimentação suplementar do rodeio é um elemento fundamental da tecnologia na produção leiteira. Além disso, seria interessante, em futuros trabalhos, utilizar dados em painel, o que incrementaria a capacidade de análise da metodologia e permitiria um melhor entendimento da evolução do setor ao longo do tempo. 


\section{Referências}

Aigner, D., Lovell, C. A. K., \& Schmidt, P. (1977). Formulation and estimation of stochastic frontier production function models. Journal of Econometrics, 6(1), 21-37.

Al-Sharafat, A. (2013). Technical efficiency of dairy farms: A stochastic frontier application on dairy farms in Jordan. The Journal of Agricultural Science, 5(3), 45.

Battese, G. E., \& Corra, G. S. (1977). Estimation of a production function model: with application to the pastoral zone of Eastern Australia. Australian Journal of Agricultural Economics, 21(3), 169-179.

Battese, G., \& Coelli, T. (1995). A Model for technical Inefficiency effects in a stochastic frontier production function for panel data. Empirical Economics. Australia., 20(2), 325-332. http://dx.doi.org/10.1007/BF01205442

Bogetoft, P., \& Otto, L. (2011). Benchmarking with DEA, SFA and R. Switzerland: Springer.

Bravo-Ureta, B. E., Moreira, V. H., Arzubi, A. A., Schilder, E. D., Álvarez, J., \& Molina, C. (2008). Cambio tecnológico y eficiencia técnica en predios lecheros de tres países del Cono Sur. Chilean Journal of Agricultural Research, 68(4), 360-367.

Cabrera, V. E., Solís, D., \& del Corral, J. (2010). Determinants of technical efficiency among dairy farms in Wisconsin. Journal of Dairy Science, 93(1), 387-393.

Chaddad, F. R. (2009). El sector lechero uruguayo en un contexto internacional: organización y estrategia social (Informe Técnico). FAO, ONU.

Coelli, T. J., Prasada Rao, D. S., O'Donnell, C. J., \& Battese, G. E. (1985). An introduction to efficiency and productivity analysis. Biometrics, 41(3), 815. http://dx.doi.org/10.2307/2531310

Dirección de Investigaciones Estadísticas Agropecuarias - DIEA. (2017). Anuario Estadístico Agropecuario. Montevideo, Uruguay: Ministerio de Ganadería, Agricultura y Pesca.

Durán, H. (2004). El camino de la lecharía: los mojones de la intensificación en sistemas pastoriles. Revista INIA Uruguay, 1, 6-9.

Farrell, M. J. (1957). The measurement of productive efficiency. Journal of the Royal Statistical Society. Series A (General), 120(3), 253-281.

Grau, C., Fossatti, M., \& Paolino, C. (1995). Eficiencia técnica y comportamiento tecnológico en establecimientos lecheros Crea. Montevideo, Uruguay: INIA.

Greene, W. H. (1980). Maximum likelihood estimation of econometric frontier functions. Journal of Econometrics, 13(1), 27-56. http://dx.doi.org/10.1016/0304-4076(80)90041-X

Guerrero, I. R., Gastaldi, L. B., \& Gatti, N. (2017). Eficiencia técnica de la lechería pampeana. Fronteras estocásticas con heterogeneidad observada y no observada. Cuadernos del CIMBAGE, 19, 87-114.

Hernández, A. (2002). El cambio técnico en el proceso de construcción de las ventajas competitivas en el sector lácteo (1975/2000) (Notas Técnicas, No. 48). Montevideo, Uruguay: Facultad de Agronomía.

Kumbhakar, S. C., Ghosh, S., \& McGuckin, J. T. (1991). A generalized production frontier approach for estimating determinants of inefficiency in U.S. dairy farms. The Journal of Business, 9(3), 279-286.

Mbaga, M., Romain, R., Larue, B., \& Lebel, L. (2003). Assessing technical efficiency of Quebec dairy farms. Canadian Journal of Agricultural Economics, 51(1), 121-137.

Meeusen, W., \& Van den Broeck, J. (1977). Efficiency estimation from Cobb-Douglas production functions with composed error. International Economic Review, 18(2), 435-444.

Nascimento, A. C. C., Lima, J. E., Braga, M. J., Nascimento, M., \& Gomes, A. P. (2012). Eficiência técnica da atividade leiteira em Minas Gerais: uma aplicação de regressão quantílica. Revista Brasileira de Zootecnia, 41(3), 783-789. http://dx.doi.org/10.1590/S1516-35982012000300043

Silva, C. S., \& Bragagnolo, C. (2018). Eficiência técnica e ambiental da pecuária leiteira na região do triângulo mineiro e alto Paranaíba. Revista de Economia e Agronegócio, 16(2), 2526-5539.

Stevenson, R. E. (1980). Likelihood functions for generalized stochastic frontier estimation. Journal of Econometrics, 13(1), 57-66. http://dx.doi.org/10.1016/0304-4076(80)90042-1

Wang, Q. (2001). A technical efficiency analysis of Pennsylvania dairy farms. Pennsylvania, USA: Departament of Agricultural Economics and Rural Sociology, Pennsylvania State University.

Watkins, K., Hristovska, T., Mazzanti, R., Wilson Junior, C., \& Schmidt, L. (2014). Measurement of technical, allocative, economic, and scale efficiency of rice production in Arkansas using data envelopment analysis. Journal of Agricultural and Applied Economics, 46(1), 89-106.

Submetido: 16/Mar/2019

Aceito: $21 /$ Jun/2020

Classificação JEL: Q12 